\title{
Revista da Estrutura de Aço - REA
}

Recebido: 04/12/2017

Aprovado: 06/03/2018

Volume 7. Número 2 (agosto/2018). p. 120-139 - ISSN 2238-9377

Revista indexada no Latindex e Diadorim/IBICT

\section{Geometric stiffness matrix for generic cross- sections}

\author{
Patrick Kherlakian ${ }^{a}$, Thiago Dias dos Santos ${ }^{b}$, Luiz Carlos Marcos Vieira Junior ${ }^{c}$, \\ Ronald D. Ziemian ${ }^{\mathrm{d}}$, Saulo José de Castro Almeida ${ }^{*}$ \\ a, b, c, e* LabMeC, Dept. of Structural Engineering, University of Campinas \\ Campinas, São Paulo, 13083-970, Brazil, patrick.kn@gmail.com, \\ santos.td@gmail.com, vieira@fec.unicamp.br, saulojca@fec.unicamp.br* \\ ${ }^{\mathrm{d}}$ Dept. of Civil and Environmental Engineering, 367 Breakiron Engineering \\ Building, Bucknell University Lewisburg, PA 17837, USA, ziemian@bucknell.edu
}

\begin{abstract}
This paper presents the derivation of a geometric stiffness matrix, which considers crosssectional warping of a generic tridimensional thin-walled member with open crosssection. Additional terms were added to the derivation previously published to take in account uniform axial deformation together with bimoment contribution. The derivation is implemented in a new software developed by the authors: Structural System Analysis, SSA, which is based on the MASTAN2 kernel and written in MATLAB. A series of examples are presented and the results are compared to the solution given by a commercial finite element software. Satisfactory agreement was found when axial and major axis loading is applied; however, when a member is loaded in the minor axis direction, the results are considerably different indicating that more research shall be carried out to accurately predict the buckling load in the minor axis.
\end{abstract}

Keywords: Stiffness Matrix, Generic Cross-Section, Warping.

\footnotetext{
${ }^{*}$ Correspondent Author
} 


\section{Introduction}

Krajcinovic (1969) following the methods developed to perform a matrix analysis of structures composed from solid beams, developed a general matrix formulation to analyze thin-walled beams. In his paper, Krajcinovic (1969) mentions: "Since the single thin-walled member is by itself statically undetermined regardless of boundary conditions, the number of redundant forces is considerably higher than for a similar structure assembled from solid beams"; based on his observation Krajcinovic (1969) developed a matrix formulation which does not take in account non-linearity and nonsymmetric cross-section.

In the following year Barsoum and Gallagher (1970) presented a set of stiffness matrices to take in account torsional stability as well as flexural-torsional stability, but the authors cautioned the reader: "The measures of solution efficacy were less satisfactory for cases where the torsional mode predominated. This factor stems from the use of a functional representation which does not satisfy the basic governing differential equation."

Yoo (1980) presents most of the development towards deriving a stiffness matrix for solving linear static problems and eigenvalue problems, however, the authors does not include in the paper the final matrices and it becomes difficult to implement such solution. Conci (1992) presents the derivation of a geometric stiffness matrix for generic cross-section, however, the digital file with the resulting stiffness matrix is illegible and the numerical analysis cannot be reproduced.

In this paper, we have revised the assumptions made by Conci (1992) and re-derived the geometric stiffness matrix for generic cross-section; we have found some mistakes, perhaps typos, which are correctly presented herein. We have also added to the derivation presented by Conci (1992) additional terms to take in account uniform axial deformation. Note that, the geometric stiffness matrix developed in this paper can be simply added to a stiffness matrix previously developed for doubly symmetric sections given in McGuire et al. (2000) and implemented in the software MASTAN2 (2016), a MATLAB based structural analysis software. We have implemented this newly developed geometric stiffness matrix into MASTAN2 (2016) and named this new software by: Structural System Analysis (SSA). SSA is primarily based on MASTAN2 (2016) 
and it is also written in MATLAB. We compared our development with a commercial finite element software: Abaqus 6.14-1.

\section{Problem Definition}

The derivation presented in this paper is based in the virtual displacement approach. In order to apply the virtual displacement approach it is necessary to know: (i) the material constitutive relationship, (ii) the strain-displacement compatibility equation, and (iii) a displacement shape function.

In the virtual displacement approach, the expression for internal work is given in terms of strain, thus the displacement function (shape function) must be differentiated. For an axial member, the strain is given by the first derivative of the longitudinal displacement, while for torsion, the "strain" is given by the rate of change of the rotation about the $x$ axis, and for bending, the "strain" or curvature is given by the second derivative of the transversal displacement. In a general format, the strain $(e)$ is given by appropriate differentiation of the shape functions vector with respect to the spatial coordinate, $\mathbf{N}^{\prime}$, multiplied by the vector of nodal point displacements, $\Delta$,

$$
e=\mathbf{N}^{\prime} \cdot \Delta .
$$

In the same manner, it is necessary to derive an expression for the internal virtual work in terms of virtual strain, $\delta e$. The virtual strain, $\delta e$, is given by the same vector of differentiated shape function, $\mathbf{N}^{\prime}$, multiplied by the virtual vector of nodal point displacements, $\delta \Delta$,

$$
\delta e=\mathbf{N}^{\prime} \cdot(\delta \mathbf{\Delta})
$$

McGuire et al. (2000) describe the principle of virtual displacement for deformable structures as: "For a deformable structure in equilibrium under the action of a system of applied forces, the external virtual work due to an admissible virtual displacement state is equal to the internal virtual work due to the same virtual displacements", which is algebraically represented by:

$$
\delta W_{e x t}=\delta W_{i n t} .
$$

Since the virtual displacements are arbitrary, the relationship between the vector of element nodal forces, $F$, and the vector of nodal point displacements, $\Delta$, is 


$$
\mathbf{F}=\mathbf{k} \boldsymbol{\Delta}
$$

where the general expression for an element stiffness matrix, $\mathbf{k}$, is

$$
\mathbf{k}=\int_{\Omega_{e}} \mathbf{N}^{\prime} E \mathbf{N}^{\prime T} d V
$$

where $\mathbf{N}^{\prime}$ and $\mathbf{N}^{\prime \top}$ are real and virtual vector of the differentiated shape function and $E$ is the relevant elastic constants.

Simple strength of materials principles for an element in pure torsion neglects resistance to cross-sectional out-of-plane warping and the torsional shearing stresses is in equilibrium with the applied torque. When longitudinal displacement is restrained the resistance to cross-sectional out-of-plane warping shall be considered; note that, in this case, the rate of twist along the length is no longer constant. This condition is known as nonuniform torsion and it can be analyzed by introducing the rate of twist, $\partial \varphi / \partial x$, which is in equilibrium with the bimoment, $B$.

Bimoment, $B$, was first introduced by Vlasov (1961) and it can be easily understood in Figure 1. Consider an axial force, $P$, applied on the tip of an I-beam, Figure 1a. Figure 1a is equivalent to superposing the effect of the axial force, Figure $1 \mathrm{~b}$, the bending moment about $z$ axis, Figure $1 c$, and the bending moment about the $y$ axis, Figure $1 d$. When summing all these components, however, the system is found to not be in equilibrium and it is necessary to add the self-equilibrated forces depicted in Figure 1e; these forces are responsible for bending each flange in an opposite direction and, therefore, warping the cross-section due to a warping moment (aka bimoment).

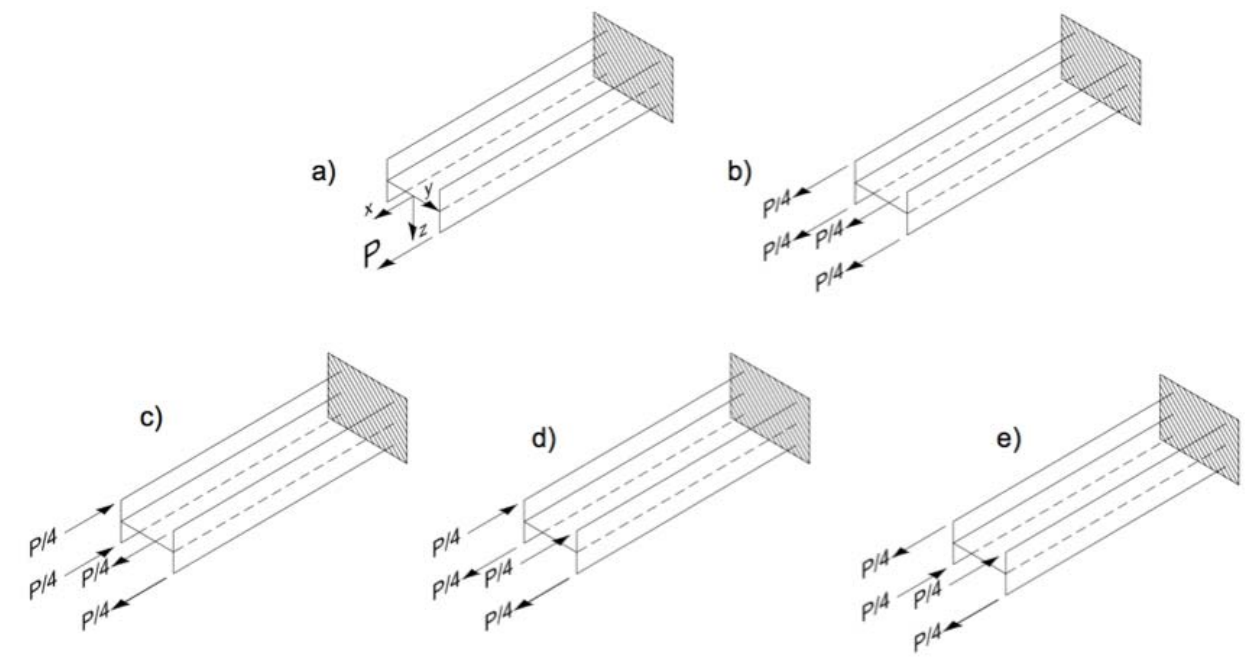

Figure 1: Equivalent system of forces 
For wide flange members, it is acceptable to admit that the bimoment corresponds to a moment of opposite direction applied to each flange multiplied by the distance between both flanges, which considerably simplifies the element stiffness equations. Traditional cold-formed steel cross-sections, however, are not usually double symmetric sections and, therefore, this assumption may lead to solutions that are not accurate.

Given that the bimoment exists, and following the nomenclature depicted in Figure 2, the normal stress is given by

$$
\sigma_{x}=+\frac{N}{A}+\frac{M_{z}}{I_{z}} y+\frac{M_{y}}{I_{y}} z+\frac{B}{I_{\omega}} \omega
$$

where,

- $\omega$ = sectorial area;

- $I_{\omega}=\mathrm{C}_{\omega}=$ warping constant.

Based on the virtual displacement principles, an updated Lagragian formulation can be linearized McGuire et al. (2000), which results in

$$
\int_{\Omega}(\mathbf{C e}): \delta \mathbf{e} d V+\int_{\Omega} \mathbf{T}: \delta \mathbf{e} d V+\int_{\Omega} \mathbf{T} \delta \boldsymbol{\eta} d V=R^{t+\Delta t},
$$

where $\mathbf{C}$ is the 4 th stress-strain tensor, $\mathbf{T}$ is the Cauchy stress tensor, $\mathbf{e}$ and $\boldsymbol{\eta}$ are defined below. The first and second integral in Eq. (7) represents the conventional elastic stiffness matrix and the forces acting on the element in the reference configuration, respectively. The last integral is of our immediate interest. The usual definition of the Green-Lagrange strains expressed in terms of the reference state can be decomposed into linear and nonlinear components, $\boldsymbol{\varepsilon}=\mathbf{e}+\boldsymbol{\eta}$, where:

$$
\mathbf{e}=\frac{1}{2}\left(\nabla \mathbf{u}+\nabla \mathbf{u}^{T}\right)
$$

and

$$
\boldsymbol{\eta}=\frac{1}{2}\left(\nabla \mathbf{u}^{T} \nabla \mathbf{u}\right),
$$

where $\mathbf{\nabla u}$ is the deformation gradient $(\mathbf{u}=\mathbf{u}(\mathrm{x}, \mathrm{y}, \mathrm{z})$ is the deformation map). For this work purpose, we need to rewrite last integral of Eq. (7) for a nonsymmetrical framework element, as shown in Figure 2. 


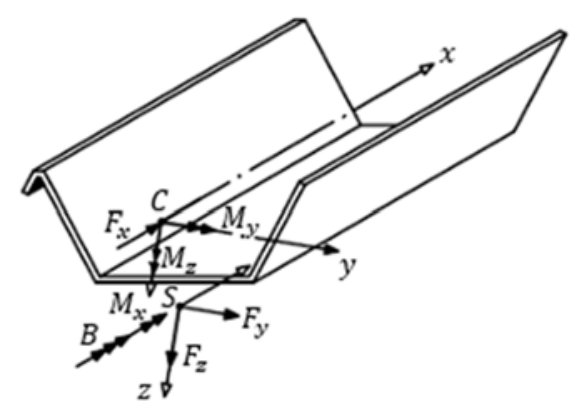

Figure 2: Internal Forces

For this framework, the stresses $\sigma_{y}, \sigma_{z}$ and $\tau_{y z}$ can be not considered. Since $\tau_{y x}=\tau_{x y}$ and $\tau_{z x}=\tau_{x z}$, the only independent stresses at any point on a cross section are $\sigma_{x}, \tau_{x y}$ and $\tau_{x z}$. Thus, the tensors $\mathbf{T}$, and $\boldsymbol{\eta}$ are reduced to:

$$
\begin{aligned}
& \mathbf{T}=\left(\sigma_{x}, \tau_{x y}, \tau_{x z}\right)^{T} \\
& \mathbf{e}=\left(e_{x}, \gamma_{x y}, \gamma_{x z}\right)^{T} \\
& \boldsymbol{\eta}=\left(\eta_{x x}, \eta_{x y}, \eta_{x z}\right)^{T}
\end{aligned}
$$

Using Eq. (10), last integral of Eq. (7) becomes:

$$
\begin{aligned}
\int_{\Omega} \mathbf{T} \delta \boldsymbol{\eta} d V & =\frac{1}{2} \int_{\Omega} \sigma_{x} \delta\left[\left(\frac{\partial u_{x}}{\partial x}\right)^{2}+\left(\frac{\partial u_{y}}{\partial x}\right)^{2}+\left(\frac{\partial u_{z}}{\partial x}\right)^{2}\right] d V \\
& +\frac{1}{2} \int_{\Omega} \tau_{x y} \delta\left[\frac{\partial u_{x}}{\partial x} \frac{\partial u_{x}}{\partial y}+\frac{\partial u_{z}}{\partial x} \frac{\partial u_{z}}{\partial y}\right] d V \\
& +\frac{1}{2} \int_{\Omega} \tau_{x z} \delta\left[\frac{\partial u_{x}}{\partial x} \frac{\partial u_{x}}{\partial z}+\frac{\partial u_{y}}{\partial x} \frac{\partial u_{y}}{\partial z}\right] d V
\end{aligned}
$$

Considering the Vlasov hypothesis of absence of shearing strain in the profile, supposing rigid cross-sectional shape and small twist angle about the shear center $\left(\Theta_{x, T}=0\right)$, as well as considered by Conci (1992), the displacement of a arbitrary point $(x, y, z)$ is given by,

$$
\begin{gathered}
u_{x}(x, y, z)=u_{x}(x)-z u_{z}^{\prime}(x)-y u_{y}^{\prime}(x)-\omega(y, z) \theta_{x}^{\prime}(x) \\
u_{y}(x, y, z)=u_{y}(x)-\left(z-z_{S}\right) \theta_{x}(x) \\
u_{z}(x, y, z)=u_{z}(x)+\left(y-y_{S}\right) \theta_{x}(x)
\end{gathered}
$$

where $u_{y}, u_{z}$ and $\theta_{x}$ are displacements above the shear center $S\left(y_{s}, z_{s}\right), u_{x}$ is the centroidal displacement $C$ and signal ' means derivation to the argument (ex. $\boldsymbol{u}_{\boldsymbol{i}}^{\prime}(\boldsymbol{x})=$ $\left.\boldsymbol{d} \boldsymbol{u}_{\boldsymbol{i}} / \boldsymbol{d} \boldsymbol{x}\right)$. Shear and center axes are shown in Figure 3. 
Conci (1992) subdivided Eq. (11) in components with terms found in doubly symmetric sections, $\boldsymbol{R}_{\boldsymbol{g}}^{S}$, and in non-symmetric cross-sections, $\boldsymbol{R}_{\boldsymbol{g}}^{G}$. Here, we also derived these two components. Following Chen and Atsuta (2007), we have the identities:

$$
\begin{aligned}
& I_{P}=I_{y}+I_{z}+\left(y_{S}^{2}+z_{S}^{2}\right) A \\
& \beta_{y}=\frac{1}{I_{y}} \int\left(z^{3}+z y^{2}\right) d A-2 z_{S} \\
& \beta_{z}=\frac{1}{I_{z}} \int\left(y^{3}+y z^{2}\right) d A-2 y_{S} \\
& \beta_{w}=\frac{1}{C_{w}} \int\left(w z^{2}+w y^{2}\right) d A
\end{aligned}
$$

Using the displacements of Eq. (12) and the coordinate system of the McGuire et al. (2000), we have the symmetric and non-symmetric components of Eq. (11), respectively:

$$
\begin{aligned}
R_{g}^{S} & =\int_{0}^{l} \frac{F_{x}}{2} \delta\left(u_{x}^{\prime 2}+u_{y}^{\prime 2}+u_{z}^{\prime 2}+\frac{I_{p}}{A} \theta_{x}^{\prime 2}\right) d x \\
& -\int_{0}^{l} M_{z} \delta\left(u_{z}^{\prime} \theta_{x}^{\prime}\right) d x \\
& -\int_{0}^{l} M_{y} \delta\left(u_{y}^{\prime} \theta_{x}^{\prime}\right) d x \\
& -\int_{0}^{l} F_{y} \delta\left(u_{x}^{\prime} u_{y}^{\prime}-u_{z}^{\prime} \theta_{x}\right) d x \\
& -\int_{0}^{l} F_{z} \delta\left(u_{x}^{\prime} u_{z}^{\prime}+u_{y}^{\prime} \theta_{x}\right) d x \\
R_{g}^{G}= & \int_{0}^{l} F_{x} \delta\left(z_{S} u_{y}^{\prime} \theta_{x}^{\prime}-y_{S} u_{z}^{\prime} \theta_{x}^{\prime}\right) d x \\
+ & \int_{0}^{l} \frac{1}{2}\left(-M_{z} \beta_{z}+M_{y} \beta_{y}+B \beta_{w}\right) \delta\left(\theta_{x}^{\prime}\right)^{2} d x \\
& -\int_{0}^{l} F_{y} \delta\left(y_{S} \theta_{x} \theta_{x}^{\prime}\right) d x \\
& -\int_{0}^{l} F_{z} \delta\left(z_{S} \theta_{x} \theta_{x}^{\prime}\right) d x
\end{aligned}
$$

The symmetric and non-symmetric geometric matrices are derived, respectively, from Eq. (14) and Eq. (15). 


\section{Derivation of Stiffness Matrix}

The derivation of the stiffness matrix was based on the following Hermite polynomials:

$$
\begin{aligned}
& \mathbf{m}_{1}=(1-\varepsilon, \varepsilon) \\
& \dot{\mathbf{m}}_{3}=\left(1-3 \varepsilon^{2}+2 \varepsilon^{3},\left(\varepsilon-2 \varepsilon^{2}+\varepsilon^{3}\right) l, 3 \varepsilon^{2}-2 \varepsilon^{3},\left(-\varepsilon^{2}+\varepsilon^{3}\right) l\right) \\
& \mathbf{m}_{3}=\left(1-3 \varepsilon^{2}+2 \varepsilon^{3},-\left(\varepsilon-2 \varepsilon^{2}+\varepsilon^{3}\right) l, 3 \varepsilon^{2}-2 \varepsilon^{3},-\left(-\varepsilon^{2}+\varepsilon^{3}\right) l\right)
\end{aligned}
$$

in which $\varepsilon=x /$ l. Given the degrees of freedom depicted in Figure 3, the following variables can be defined as:

$$
\begin{aligned}
& \mathbf{M}_{z}=\left(-M_{z A}, M_{z B}\right) \\
& \mathbf{M}_{y}=\left(-M_{y A}, M_{y B}\right) \\
& \mathbf{u}_{x}=\left(u_{x A}, u_{x B}\right) \\
& \mathbf{u}_{y}=\left(u_{y A}, \theta_{z A}, u_{y B}, \theta_{z B}\right) \\
& \mathbf{u}_{z}=\left(u_{z A},-\theta_{y A}, u_{z B},-\theta_{y B}\right) \\
& \boldsymbol{\theta}_{x}=\left(\theta_{x A}, \theta_{x A}^{\prime}, \theta_{x B}, \theta_{x B}^{\prime}\right)
\end{aligned}
$$

Using Eq. (16) and Eq. (17), the internal forces, displacements and rotation are rewritten using tensorial notation:

$$
\begin{aligned}
& F_{x}=F_{x B} \\
& F_{y}=-\left(M_{z A}+M_{z B}\right) / l \\
& F_{z}=\left(M_{y A}+M_{y B}\right) / l \\
& M_{z}=\mathbf{m}_{1} \cdot \mathbf{M}_{z} \\
& M_{y}=\mathbf{m}_{1} \cdot \mathbf{M}_{y} \\
& u_{x}=\mathbf{m}_{1} \cdot \mathbf{u}_{x} \\
& u_{y}=\mathbf{m}_{3} \cdot \mathbf{u}_{y} \\
& u_{z}=\mathbf{m}_{3} \cdot \mathbf{u}_{z} \\
& \theta_{x}=\dot{\mathbf{m}}_{3} \cdot \boldsymbol{\theta}_{x}
\end{aligned}
$$

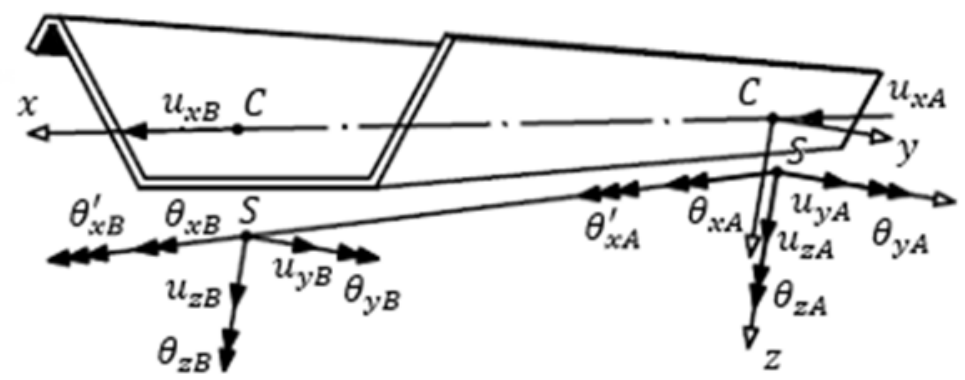

Figure 3: Degrees of freedom for a generic section. 


\subsection{Derivation of Stiffness Matrix for Symmetric Cross-sections}

Using Eq. (18) in the Eq. (14), we have:

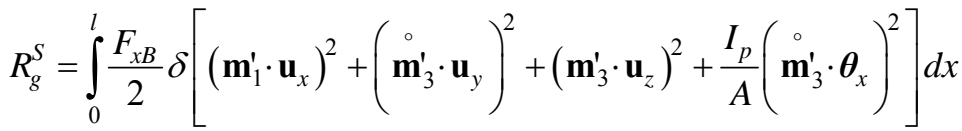

$$
\begin{aligned}
& -\int_{0}^{l}\left(\mathbf{m}_{1} \cdot \mathbf{M}_{z}\right) \delta\left[\left(\mathbf{m}_{3}^{\prime} \cdot \mathbf{u}_{z}\right)\left(\stackrel{\circ}{\mathbf{m}_{3}^{\prime}} \cdot \boldsymbol{\theta}_{x}\right)\right] d x \\
& -\int_{0}^{l}\left(\mathbf{m}_{1} \cdot \mathbf{M}_{y}\right) \delta\left[\left(\stackrel{\circ}{\mathbf{m}_{3}^{\prime} \cdot \mathbf{u}_{y}}\right)\left(\stackrel{\circ}{\mathbf{m}_{3}^{\prime} \cdot \boldsymbol{\theta}_{x}}\right)\right] d x
\end{aligned}
$$

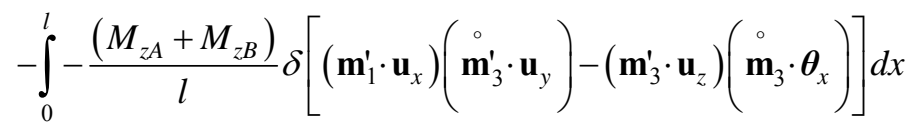

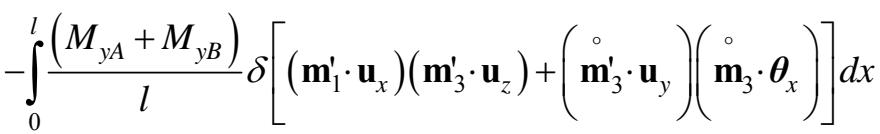

The following tensor properties (Gurtin, 1982) are applied in the next step:

$$
\begin{gathered}
(a \cdot u)(b \cdot v)=(a \otimes b) \cdot(u \otimes v), \\
S \cdot(u \otimes v)=(S v) \cdot u=v \cdot S^{T} u \\
(a \otimes b)^{T}=(b \otimes a)
\end{gathered}
$$

Using Eq. (20), Eq. (21), Eq. (22), grouping some parts and applying the virtual operator $\delta$ we finally have the symmetric cross-section geometric matrix:

$$
\begin{aligned}
& R_{g}^{S}=\delta \mathbf{u}_{x} \cdot\left\{F_{x B}\left[\int_{0}^{l}\left(\mathbf{m}_{1}^{\prime} \otimes \mathbf{m}_{1}^{\prime}\right) d x\right]\right\} \mathbf{u}_{x} \\
& +\delta \mathbf{u}_{y} \cdot\left\{F_{x B}\left[\int_{0}^{l}\left(\stackrel{\circ}{\dot{m}_{3}^{\prime} \otimes}{\stackrel{\circ}{\mathbf{m}^{\prime}}}_{3}\right) d x\right]\right\} \mathbf{u}_{y} \\
& +\delta \mathbf{u}_{z} \cdot\left\{F_{x B}\left[\int_{0}^{l}\left(\mathbf{m}_{3}^{\prime} \otimes \mathbf{m}_{3}^{\prime}\right) d x\right]\right\} \mathbf{u}_{z} \\
& +\delta \boldsymbol{\theta}_{x} \cdot\left\{F_{x B} \frac{I_{p}}{A}\left[\int_{0}^{l}\left(\stackrel{\circ}{\mathbf{m}_{3}^{\prime} \otimes \dot{m}_{3}^{\prime}}\right) d x\right]\right\} \boldsymbol{\theta}_{x} \\
& -\delta \mathbf{u}_{z} \cdot\left\{\int_{0}^{l}\left(\mathbf{m}_{1} \cdot \mathbf{M}_{z}\right)\left(\mathbf{m}_{3}^{\prime} \otimes{\stackrel{\circ}{\mathbf{m}^{\prime}}}_{3}\right) d x\right\} \boldsymbol{\theta}_{x}
\end{aligned}
$$

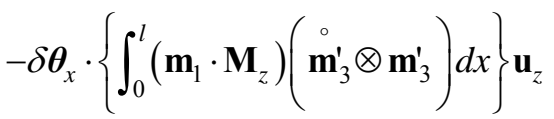




$$
\begin{aligned}
& -\delta \mathbf{u}_{y} \cdot\left\{\int_{0}^{l}\left(\mathbf{m}_{1} \cdot \mathbf{M}_{y}\right)\left(\stackrel{\circ}{\mathbf{m}_{3}^{\prime} \otimes} \stackrel{\circ}{\mathbf{m}_{3}^{\prime}}\right) d x\right\} \boldsymbol{\theta}_{x} \\
& -\delta \boldsymbol{\theta}_{x} \cdot\left\{\int_{0}^{l}\left(\mathbf{m}_{1} \cdot \mathbf{M}_{y}\right)\left(\stackrel{\circ}{\dot{\mathbf{m}}_{3}^{\prime} \otimes} \stackrel{\circ}{\mathbf{m}_{3}^{\prime}}\right) d x\right\} \mathbf{u}_{y} \\
& +\delta \mathbf{u}_{x} \cdot\left\{\frac{\left(M_{z A}+M_{z B}\right)}{l}\left[\int_{0}^{l}\left(\mathbf{m}_{1}^{\prime} \otimes \stackrel{\circ}{\mathbf{m}_{3}^{\prime}}\right) d x\right]\right\} \mathbf{u}_{y}
\end{aligned}
$$

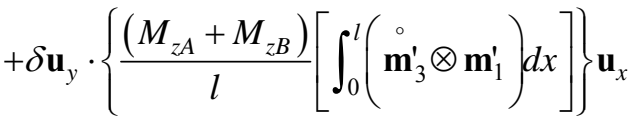

$$
\begin{aligned}
& -\delta \mathbf{u}_{z} \cdot\left\{\frac{\left(M_{z A}+M_{z B}\right)}{l}\left[\int_{0}^{l}\left(\mathbf{m}_{3}^{\prime} \otimes \stackrel{\circ}{\mathbf{m}}_{3}\right) d x\right]\right\} \boldsymbol{\theta}_{x}
\end{aligned}
$$

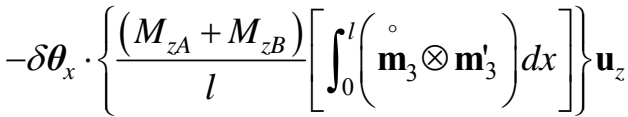

$$
\begin{aligned}
& -\delta \mathbf{u}_{x} \cdot\left\{\frac{\left(M_{y A}+M_{y B}\right)}{l}\left[\int_{0}^{l}\left(\mathbf{m}_{1}^{\prime} \otimes \mathbf{m}_{3}^{\prime}\right) d x\right]\right\} \mathbf{u}_{z} \\
& -\delta \mathbf{u}_{z} \cdot\left\{\frac{\left(M_{y A}+M_{y B}\right)}{l}\left[\int_{0}^{l}\left(\mathbf{m}_{3}^{\prime} \otimes \mathbf{m}_{1}^{\prime}\right) d x\right]\right\} \mathbf{u}_{x} \\
& -\delta \mathbf{u}_{y} \cdot\left\{\frac{\left(M_{y A}+M_{y B}\right)}{l}\left[\int_{0}^{l}\left(\stackrel{\circ}{\dot{m}_{3}^{\prime} \otimes} \stackrel{\circ}{\mathbf{m}}_{3}\right) d x\right]\right\} \boldsymbol{\theta}_{x}
\end{aligned}
$$

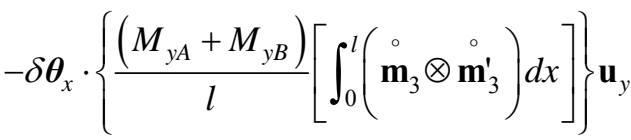

\subsection{Derivation of Stiffness Matrix for Non-Symmetric Cross-sections}

Similar to symmetric cross-sections, applying Eq. (18) in the Eq. (15), and using the tensor properties Eq. (20), Eq. (21), Eq. (22), and grouping some terms, we have:

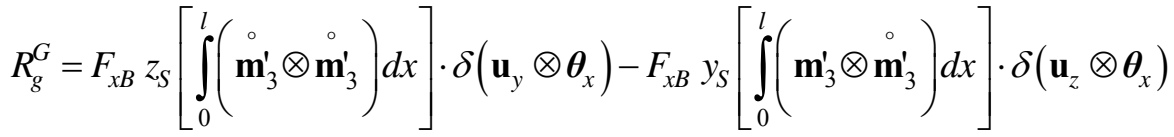

$$
\begin{aligned}
& +\frac{1}{2}\left\{\int_{0}^{l}\left[-\left(\mathbf{m}_{1} \cdot \mathbf{M}_{z}\right) \beta_{z}+\left(\mathbf{m}_{1} \cdot \mathbf{M}_{y}\right) \beta_{y}+\left(\mathbf{m}_{1} \cdot \mathbf{B}\right) \beta_{w}\right]\left(\stackrel{\circ}{\mathbf{m}_{3}^{\prime} \otimes \stackrel{\circ}{\mathbf{m}_{3}^{\prime}}}\right) d x\right\} \cdot \delta\left(\boldsymbol{\theta}_{x} \otimes \boldsymbol{\theta}_{x}\right) \\
& +\frac{\left(M_{\mathrm{zA}}+M_{z B}\right)}{l} y_{S}\left[\int_{0}^{l}\left(\dot{\circ}_{3} \otimes \dot{\mathbf{m}}_{3}^{\prime}\right) d x\right] \cdot \delta\left(\boldsymbol{\theta}_{x} \otimes \boldsymbol{\theta}_{x}\right) \\
& -\frac{\left(M_{y A}+M_{y B}\right)}{l} z_{S}\left[\int_{0}^{l}\left(\dot{\circ}_{3} \otimes \dot{m}_{3}^{\prime}\right) d x\right] \cdot \delta\left(\boldsymbol{\theta}_{x} \otimes \boldsymbol{\theta}_{x}\right)
\end{aligned}
$$

Applying the virtual operator $\delta$ and using the tensor properties, we finally have the nonsymmetric cross-section geometric matrix: 


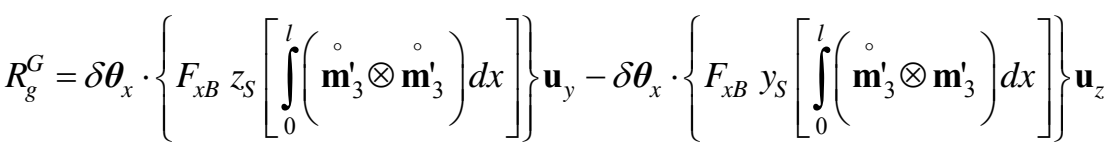

$$
\begin{aligned}
& +\delta \mathbf{u}_{y} \cdot\left\{F_{x B} z_{S}\left[\int_{0}^{l}\left(\stackrel{\circ}{\mathbf{m}_{3}^{\prime} \otimes \dot{m}_{3}^{\prime}}\right) d x\right]\right\} \boldsymbol{\theta}_{x}-\delta \mathbf{u}_{z} \cdot\left\{F_{x B} y_{S}\left[\int_{0}^{l}\left(\mathbf{m}_{3}^{\prime} \otimes \stackrel{\circ}{\mathbf{m}_{3}^{\prime}}\right) d x\right]\right\} \boldsymbol{\theta}_{x} \\
& +\delta \boldsymbol{\theta}_{x} \cdot\left\{\int_{0}^{l}\left[-\left(\mathbf{m}_{1} \cdot \mathbf{M}_{z}\right) \beta_{z}+\left(\mathbf{m}_{1} \cdot \mathbf{M}_{y}\right) \beta_{y}+\left(\mathbf{m}_{1} \cdot \mathbf{B}\right) \beta_{w}\right]\left(\stackrel{\circ}{\mathbf{m}_{3}^{\prime} \otimes \dot{m}_{3}^{\prime}}\right) d x\right\} \boldsymbol{\theta}_{x}
\end{aligned}
$$

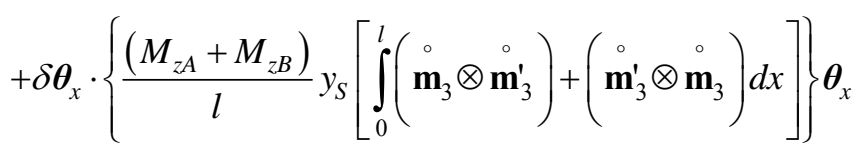

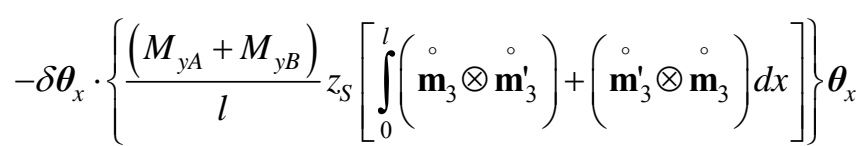

Based on the development depicted previously, $K_{G, \text { Symmetric }}$ (from Eq. 23) and

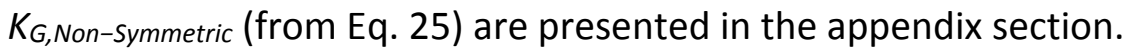

\section{Examples}

In order to validate the symmetric and non-symmetric stiffness matrices, we implemented them both in SSA. Six examples will be given, where the first ten elastic buckling modes were computed and compared with the obtained in the software Abaqus 6.14-1, where the element B31OS was used. B31OS is a tridimensional open section beam element that uses linear interpolation, Abaqus (2014). Yoo (1980) reports that elastic buckling loads converge to the same value once the member is discretized into 16 elements; thus, all examples herein are conservatively modeled with 32 equal size elements. Two different loads were applied in all examples studied herein: unitary axial load to the center of gravity, $F_{x}$, and loads applied to the shear center in the major cross-section axis direction. The effect of loading applied to the minor axis has small practical importance, but the authors have found that further studies have to be conducted in the topic, since, in our examples, it was not found a satisfactory agreement when loading is applied in the minor axis, for both: symmetric and non-symmetric crosssections. 


\subsection{Example 1}

A beam with length of $7,320 \mathrm{~mm}$ was analyzed. It has a symmetric I cross-section with web of $508 \mathrm{~mm}$ and flanges of $305 \mathrm{~mm}$. The cross-section thickness was defined with 13 $\mathrm{mm}$. It was used an isotropic material of Young's Modulus of $200 \mathrm{GPa}$ and Poisson's Ratio of 0.25 . The boundary conditions presented in Figure 4 are considered and two load cases are applied: a concentrated axial load, $F_{x}$, and a concentrated load at mid-length in the direction of the $y$-axis, $F_{y}$. The results are tabulated in Table 1 . The first ten buckling loads calculated by Abaqus and SSA had an average difference of $5.8 \%$ and $5.1 \%$ for $F_{x}$ and $F_{y}$ loads, respectively, which is considered a satisfactory agreement between both models.

$$
\begin{gathered}
u_{x}(0)=u_{y}(0)=u_{y}(\mathrm{~L})=u_{z}(0)=u_{z}(\mathrm{~L})=0 \\
\theta_{x}(0)=\theta_{x}(L)=\theta_{y}(0)=\theta_{y}(L)=\theta_{z}(0)=\theta_{z}(L)=0 \\
\theta_{x}^{\prime}(0)=\theta_{x}^{\prime}(\mathrm{L})=0
\end{gathered}
$$

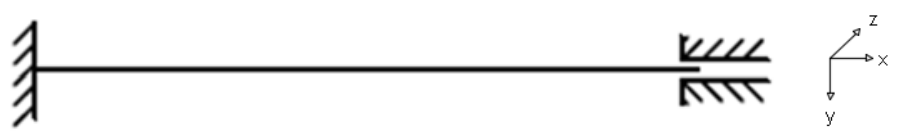

Figure 4: Boundary Conditions for Example 1.

Table 1: Buckling Loads for Example 1.

\begin{tabular}{ccccccc}
\hline Modes & SSA & Abaqus & Abaqus/SSA & SSA & Abaqus & Abaqus/SSA \\
& $F_{x}(N)$ & $F_{x}(N)$ & & $F_{y}(N)$ & $F_{y}(N)$ & \\
\hline 1 & 9058576 & 8915390 & 0.98 & 2802455 & 2754150 & 0.98 \\
2 & 13209187 & 13179900 & 1.00 & 11195287 & 10867000 & 0.97 \\
3 & 18531689 & 17873900 & 0.96 & 23916965 & 22808200 & 0.95 \\
4 & 25630697 & 25353800 & 0.99 & 40690609 & 39223300 & 0.96 \\
5 & 36235416 & 34044800 & 0.94 & 58393375 & 55575600 & 0.95 \\
6 & 48844502 & 47798500 & 0.98 & 77008669 & 73774500 & 0.96 \\
7 & 54779359 & 49849210 & 0.91 & 86351502 & 78237100 & 0.91 \\
8 & 73160032 & 70522700 & 0.96 & 98747694 & 91291400 & 0.92 \\
9 & 81540406 & 71203700 & 0.87 & 125752176 & 117642000 & 0.94 \\
10 & 96317211 & 79893900 & 0.83 & 164743938 & 156054000 & 0.95 \\
\hline \hline
\end{tabular}

\subsection{Example 2}

The problem examined in this subsection presents the same cross-section depicted in Figure $5 a$ with thickness of $2 \mathrm{~mm}$ and beam length of 2,000 mm. The material is considered isotropic and has a Young's Modulus of 205 GPa and a Poisson's Ratio of 0.30. The boundary conditions are presented in Figure $5 \mathrm{~b}$ and a concentrated axial load, $F_{x}$, and the effect of a distributed load, $q_{y}$ is analyzed. The results of computer analyses are tabulated in Table 2. The average difference between Abaqus and SSA's buckling 
loads was $2.2 \%$ and $3 \%$ for $F_{x}$ and $q_{y}$ loads, respectively. The models lead to a satisfactory agreement for both applied loads.
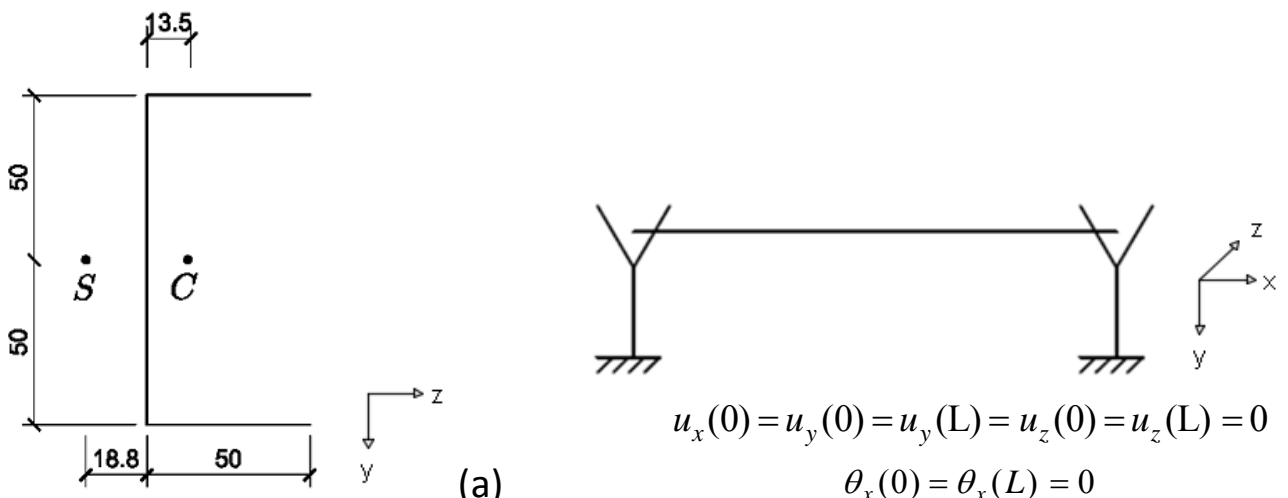
$\theta_{X}(0)=\theta_{X}(L)=0$

Figure 5: (a) Channel section, units in $\mathrm{mm}$ and (b) Boundary conditions for example 2.

Table 2: Buckling Loads for Example 2.

\begin{tabular}{ccccccc}
\hline \hline Modes & SSA & $\begin{array}{c}\text { Abaqus } \\
F_{x}(N)\end{array}$ & Abaqus/SSA & $\begin{array}{c}\text { SSA } \\
q_{y}(\mathrm{~N} / \mathrm{mm})\end{array}$ & $\begin{array}{c}\text { Abaqus } \\
q_{y}(\mathrm{~N} / \mathrm{mm})\end{array}$ & Abaqus/SSA \\
\hline 1 & 44015 & 42789 & 0.97 & 6.02 & 6.03 & 1.00 \\
2 & 52689 & 52658 & 1.00 & 24.97 & 25.03 & 1.00 \\
3 & 136347 & 132934 & 0.97 & 58.31 & 58.65 & 1.01 \\
4 & 210758 & 210263 & 1.00 & 105.94 & 107.10 & 1.01 \\
5 & 289980 & 283458 & 0.98 & 167.89 & 170.87 & 1.02 \\
6 & 474209 & 471700 & 0.99 & 244.18 & 250.62 & 1.03 \\
7 & 505043 & 495319 & 0.98 & 334.83 & 347.11 & 1.04 \\
8 & 533749 & 578936 & 1.08 & 439.89 & 461.48 & 1.05 \\
9 & 781571 & 769679 & 0.98 & 559.41 & 594.81 & 1.06 \\
10 & 843056 & 835111 & 0.99 & 693.50 & 749.00 & 1.08 \\
\hline \hline
\end{tabular}

\subsection{Example 3}

A beam of 12,000 mm length with cross-section depicted in Figure 6 was tested with the same boundary conditions of Example 2. A concentrated axial load, $F_{x}$, and the effect of a distributed load, $\mathrm{q}_{\mathrm{y}}$ is analyzed. This cross-section was based on Palermo (1985). The material used has Young's Modulus of 205.9 GPa and Poisson's Ratio of 0.3125. The thickness was $10 \mathrm{~mm}$. SSA and Abaqus results are compared in Table 3. While there is a satisfactory agreement when axial $F_{x}$ loading is applied, when $q_{y}$ is applied the difference is as high as $58 \%$ for the 10 th mode. This difference occurs only in cases where there is not a single symmetry axis. Note that for the first buckling mode, usually the mode with most practical interest in design calculation, a difference of only $5 \%$ has been found. $A$ possible reason for this divergence is due to the considerations taken in account while deriving the stiffness matrix implemented in Abaqus. According to the commercial software documentation theory guide (Abaqus, 2014), the derivation of the stiffness 
matrix of element B310S considers transverse shear strain while our derivation implemented in SSA and presented herein does not consider it.

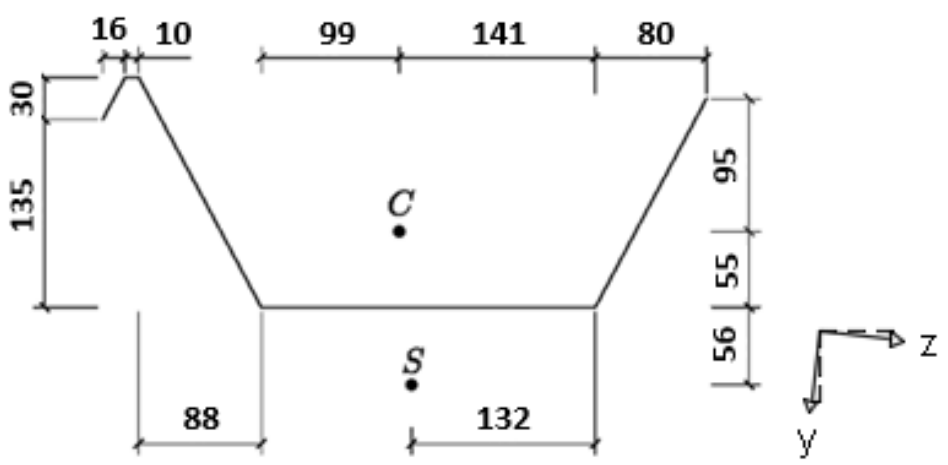

Figure 6: Gutter beam cross-section. Units in $\mathrm{mm}$.

Table 3: Buckling Loads for Example 3.

\begin{tabular}{|c|c|c|c|c|c|c|}
\hline Modes & $\begin{array}{c}\text { SSA } \\
F_{x}(N)\end{array}$ & $\begin{array}{c}\text { Abaqus } \\
F_{x}(N)\end{array}$ & Abaqus/SSA & $\begin{array}{c}\mathrm{SSA} \\
q_{y}(\mathrm{~N} / \mathrm{mm})\end{array}$ & $\begin{array}{c}\text { Abaqus } \\
q_{y}(\mathrm{~N} / \mathrm{mm})\end{array}$ & Abaqus/SSA \\
\hline 1 & 270075 & 270173 & 1.00 & 4.02 & 4.32 & 1.05 \\
\hline 2 & 475818 & 475720 & 1.00 & 9.91 & 11.47 & 1.16 \\
\hline 3 & 609581 & 610365 & 1.00 & 17.46 & 21.28 & 1.22 \\
\hline 4 & 822679 & 827485 & 1.01 & 27.16 & 34.62 & 1.27 \\
\hline 5 & 1108739 & 1124430 & 1.01 & 39.23 & 51.88 & 1.32 \\
\hline 6 & 1129922 & 1132177 & 1.00 & 53.84 & 73.55 & 1.37 \\
\hline 7 & 1473841 & 1512871 & 1.03 & 71.00 & 100.03 & 1.41 \\
\hline 8 & 1919161 & 2001439 & 1.04 & 90.71 & 132.39 & 1.46 \\
\hline 9 & 2445190 & 2530900 & 1.04 & 112.78 & 171.62 & 1.52 \\
\hline 10 & 2519132 & 2600233 & 1.03 & 138.27 & 218.69 & 1.58 \\
\hline
\end{tabular}

\subsection{Example 4}

The same beam of Example 2 is analyzed, however, with the boundary conditions used in Example 1. A concentrated axial load, $F_{x}$, and the effect of a distributed load, $q_{y}$, is analyzed. The results are presented in Table 4. Both models lead to similar results: the average difference is $1.2 \%$ for both $\mathrm{F}_{\mathrm{x}}$ and $\mathrm{q}_{\mathrm{y}}$ loads.

Table 4: Buckling Loads for Example 4.

\begin{tabular}{ccccccc}
\hline \hline Modes & SSA & Abaqus & Abaqus/SSA & $\begin{array}{c}\text { SSA } \\
q_{y}(N / m m)\end{array}$ & $\begin{array}{c}\text { Abaqus } \\
q_{y}(N / m m)\end{array}$ & Abaqus/SSA \\
\hline 1 & 136347 & 133236 & 0.98 & 48.81 & 48.59 & 1.00 \\
2 & 210758 & 210263 & 1.00 & 146.86 & 145.84 & 0.99 \\
3 & 264879 & 259305 & 0.98 & 319.20 & 316.53 & 0.99 \\
4 & 431160 & 428601 & 0.99 & 548.49 & 550.83 & 1.00 \\
5 & 505043 & 496347 & 0.98 & 777.35 & 787.00 & 1.01 \\
6 & 756596 & 746114 & 0.99 & 1044.83 & 1082.60 & 1.04 \\
7 & 843056 & 835111 & 0.99 & 1275.30 & 1277.20 & 1.00 \\
8 & 1119612 & 1110200 & 0.99 & 1488.03 & 1487.50 & 1.00 \\
9 & 1274501 & 1254870 & 0.98 & 1701.29 & 1725.40 & 1.01 \\
10 & 1494287 & 1489060 & 1.00 & 2068.08 & 2158.60 & 1.04 \\
\hline \hline
\end{tabular}




\subsection{Example 5}

The same beam length and cross-section of Example 3, however, with boundary conditions presented in Example 1 was analyzed. A concentrated axial load, $F_{x}$, and a distributed load, $q_{y}$, were applied. Results are shown in Table 5. The mean buckling load difference for $F_{x}$ load is $3.6 \%$, which corresponds to a satisfactory agreement. For the distributed load, $\mathrm{q}_{\mathrm{y}}$, one can note larger difference: mean difference of $12.7 \%$, maximum difference of $26 \%$ for the 10 th mode. For the first mode, Abaqus and SSA generate the same buckling load. We understand that this difference can be explained by the same comment delineated in Example 3: consideration of transverse shear strain in the Abaqus geometric stiffness derivation. One can note the difference shown in this example for $q_{y}$ load is smaller than the difference observed In Example 3. We associate this differences to the constraints imposed in this example.

Table 5: Example 5 Buckling Loads

\begin{tabular}{ccccccc}
\hline \hline Modes & SSA & Abaqus & Abaqus/SSA & $\begin{array}{c}\text { SSA } \\
q_{y}(\mathrm{~N} / \mathrm{mm})\end{array}$ & $\begin{array}{c}\text { Abaqus } \\
q_{y}(\mathrm{~N} / \mathrm{mm})\end{array}$ & Abaqus/SSA \\
\hline 1 & 609581 & 610366 & 1.00 & 19.6 & 19.6 & 1.00 \\
2 & 788847 & 792966 & 1.01 & 46.1 & 52.0 & 1.12 \\
3 & 1108740 & 1124430 & 1.01 & 87.3 & 100.0 & 1.14 \\
4 & 1129922 & 1132178 & 1.00 & 143.2 & 163.8 & 1.14 \\
5 & 1440891 & 1477960 & 1.03 & 201.0 & 229.5 & 1.14 \\
6 & 1919161 & 2001439 & 1.04 & 274.6 & 312.8 & 1.14 \\
7 & 2291618 & 2300836 & 1.00 & 341.3 & 381.5 & 1.12 \\
8 & 2412338 & 2563458 & 1.06 & 426.6 & 456.0 & 1.07 \\
9 & 3052320 & 3321905 & 1.09 & 464.8 & 527.6 & 1.14 \\
10 & 3707796 & 4142329 & 1.12 & 519.8 & 653.1 & 1.26 \\
\hline \hline
\end{tabular}

\section{Conclusion}

A geometric stiffness matrix, which considers cross-sectional warping of a generic crosssection, is presented herein. The geometric stiffness matrix developed herein was implemented in MASTAN2 (2016), which lead to the creation of a new software Structural System Analysis, SSA; both software were developed in MATLAB. Additional terms were added to the derivation presented by Conci (1992) to take in account uniform axial deformation. The results are compared to commercial finite element software, Abaqus 6.14-1, and it was found that there is a satisfactory agreement when axial $F_{x}$ and distributed $q_{y}$ loads are applied for the selected examples The authors, 
however, show that Abaqus models did not lead to results similar to the results presented herein when a member is loaded in the minor axis direction. These differences occur only in cases where there is not a single symmetry axis. Nevertheless, for the first buckling mode, usually the mode with most practical interest in design analysis, a difference of only $5 \%$ has been found. The derivation of the stiffness matrix of Abaqus element B31OS considering transverse shear strain is a potential reason for this divergence since our derivation does not consider it. Although loading in minor axis is not usual, the authors recommend that more research shall be carried out to accurately predict the buckling load when the element is loaded in the minor axis.

\section{References}

ABAQUS (2014). 6.14 Documentation. Dassault Systemes Simulia Corporation

BARSOUM, R. S. and GALLAGHER, R. H. (1970). Finite element analysis of torsional and torsionalflexural stability problems. Int. J. Numer. Meth. Engng, 3(2): 335-352.

CHEN, W. and ATSUTA, T. (2007). Theory of Beam-Columns, Volume 2: Space Behavior and Design. J. Ross Publishing.

CONCI, A. (1992). Stiffness Matrix for Nonlinear Analysis of Thin-Walled Frames. J. Eng. Mech., 118(9): 1859-1875.

GURTIN, M. E. (1982). An Introduction to Continuum Mechanics. Academic Press, USA.

KRAJCINOVIC, D. (1969). A consistent discrete elements technique for thinwalled assemblages. Int. J. Solids Struct., 5(7): 639-662.

MCGUIRE, W., GALLANGER, R. H. and ZIEMIAN, R. D. (2000). Matrix Structural Analysis, 2nd edn. John Wiley \& Sons, USA.

PALERMO, L. (1985). Esforços de flexão e flexo-torção em teoria de 2a. ordem: automatizacão do cálculo. Master's Thesis in Portuguese, EESC-USP, Sao Paulo, Brazil.

VLASOV, V. Z. (1961). Thin-Walled Elastic Beams, 2nd edn. National Science Foundation, Washington, D.C., USA.

YOO, C. H. (1980). Bimoment contribution to stability of thin-walled assemblages. Comput. \& Struct., 11(5): 465-471.

ZIEMIAN, R. D. and MCGUIRE W. MASTAN 2. Version 3.5. 2016. Available at:

<http://www.mastan2.com/download.html> 


\section{Appendix A. Geometric Stiffness Matrix}

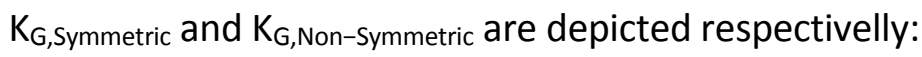

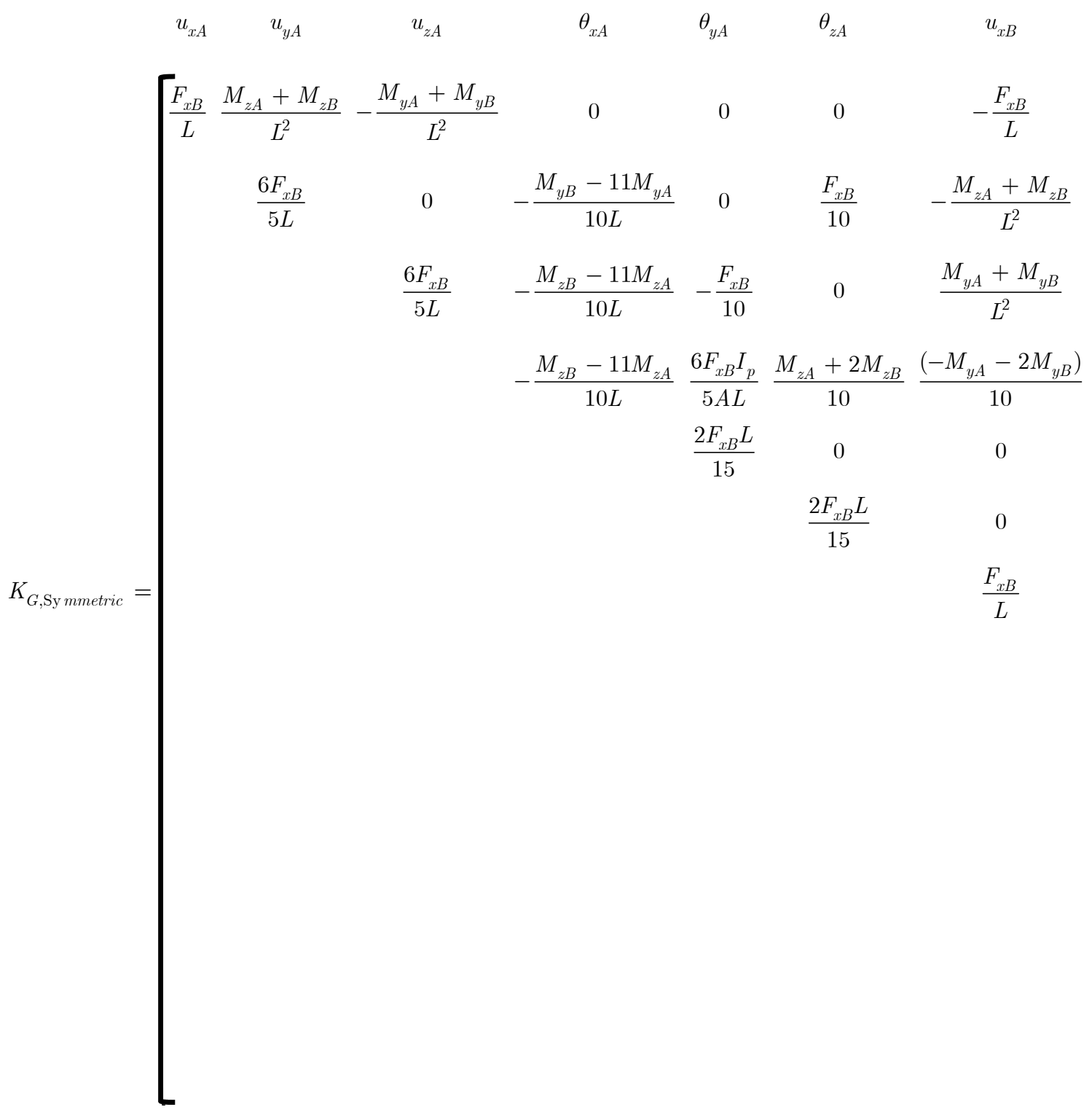




$$
\begin{aligned}
& \begin{array}{cccccc}
u_{y B} & u_{z B} & \theta_{y B} & \theta_{z B} & \theta_{x A}^{\prime} & \theta_{x B}^{\prime}
\end{array} \\
& \begin{array}{ccccccc}
-\frac{M_{z A}+M_{z B}}{L^{2}} & \frac{M_{y A}+M_{y B}}{L^{2}} & 0 & 0 & 0 & 0 & 0 \\
-\frac{6 F_{x B}}{5 L} & 0 & -\frac{M_{y A}-11 M_{y B}}{10 L} & 0 & \frac{F_{x B}}{10} & \frac{M_{y A}}{10} & -\frac{M_{y B}}{10} \\
0 & -\frac{6 F_{x B}}{5 L} & \frac{M_{z A}-11 M_{z B}}{10 L} & -\frac{F_{x B}}{10} & 0 & \frac{M_{z A}}{10} & -\frac{M_{z B}}{10}
\end{array} \\
& \frac{M_{y B}-11 M_{y A}}{10 L} \frac{M_{z B}-11 M_{z A}}{10 L} \quad-\frac{6 F_{x B} I_{p}}{5 A L} \quad \frac{\left(-2 M_{z A}-M_{z B}\right)}{10} \quad \frac{\left(2 M_{y A}+M_{y B}\right)}{10} \quad \frac{F_{x B} I_{p}}{10 A} \quad \frac{F_{x B} I_{p}}{10 A} \\
& 0 \quad \frac{F_{x B}}{10} \quad \frac{\left(-M_{z A}-2 M_{z B}\right)}{10} \quad-\frac{\left(F_{x B} L\right)}{30} \quad 0 \quad \frac{\mathrm{L}\left(3 M_{z B}-M_{z A}\right)}{30} \quad \frac{\mathrm{L} M_{z A}}{30} \\
& -\frac{F_{x B}}{10} \quad 0 \quad \frac{\left(M_{y A}+2 M_{y B}\right)}{10} \quad 0 \quad-\frac{\left(F_{x B} L\right)}{30} \quad \frac{\mathrm{L}\left(3 M_{y A}-M_{y B}\right)}{30} \quad-\frac{\left(\mathrm{L} M_{y A}\right)}{30} \\
& \begin{array}{lllllll}
\frac{M_{z A}+M_{z B}}{L^{2}}-\frac{M_{y A}+M_{y B}}{L^{2}} & 0 & 0 & 0 & 0 & 0
\end{array}
\end{aligned}
$$

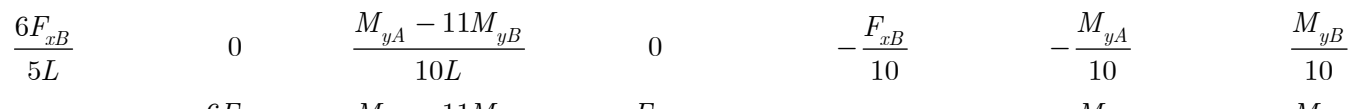

$$
\begin{aligned}
& \frac{6 F_{x B}}{5 L} \quad \frac{M_{z A}-11 M_{z B}}{10 L} \quad \frac{F_{x B}}{10} \quad 0 \quad-\frac{M_{z A}}{10} \quad \frac{M_{z B}}{10} \\
& \frac{6 F_{x B} I_{p}}{5 A L} \quad \frac{\left(2 M_{z A}+M_{z B}\right)}{10} \frac{\left(-2 M_{y A}-M_{y B}\right)}{10}-\frac{F_{x B} I_{p}}{10 A} \quad-\frac{F_{x B} I_{p}}{10 A} \\
& \frac{2 F_{x B} L}{15} \quad 0 \quad-\frac{L M_{z B}}{30} \quad-\frac{\mathrm{L}\left(M_{z A}-3 M_{z B}\right)}{30} \\
& \frac{2 F_{x B} L}{15} \quad \frac{L M_{y B}}{30} \quad \frac{\mathrm{L}\left(M_{y A}-3 M_{y B}\right)}{30} \\
& \frac{2 F_{x B} I_{p} L}{15 A} \quad-\frac{F_{x B} I_{p} L}{30 A} \\
& \frac{2 F_{x B} I_{p} L}{15 A}
\end{aligned}
$$




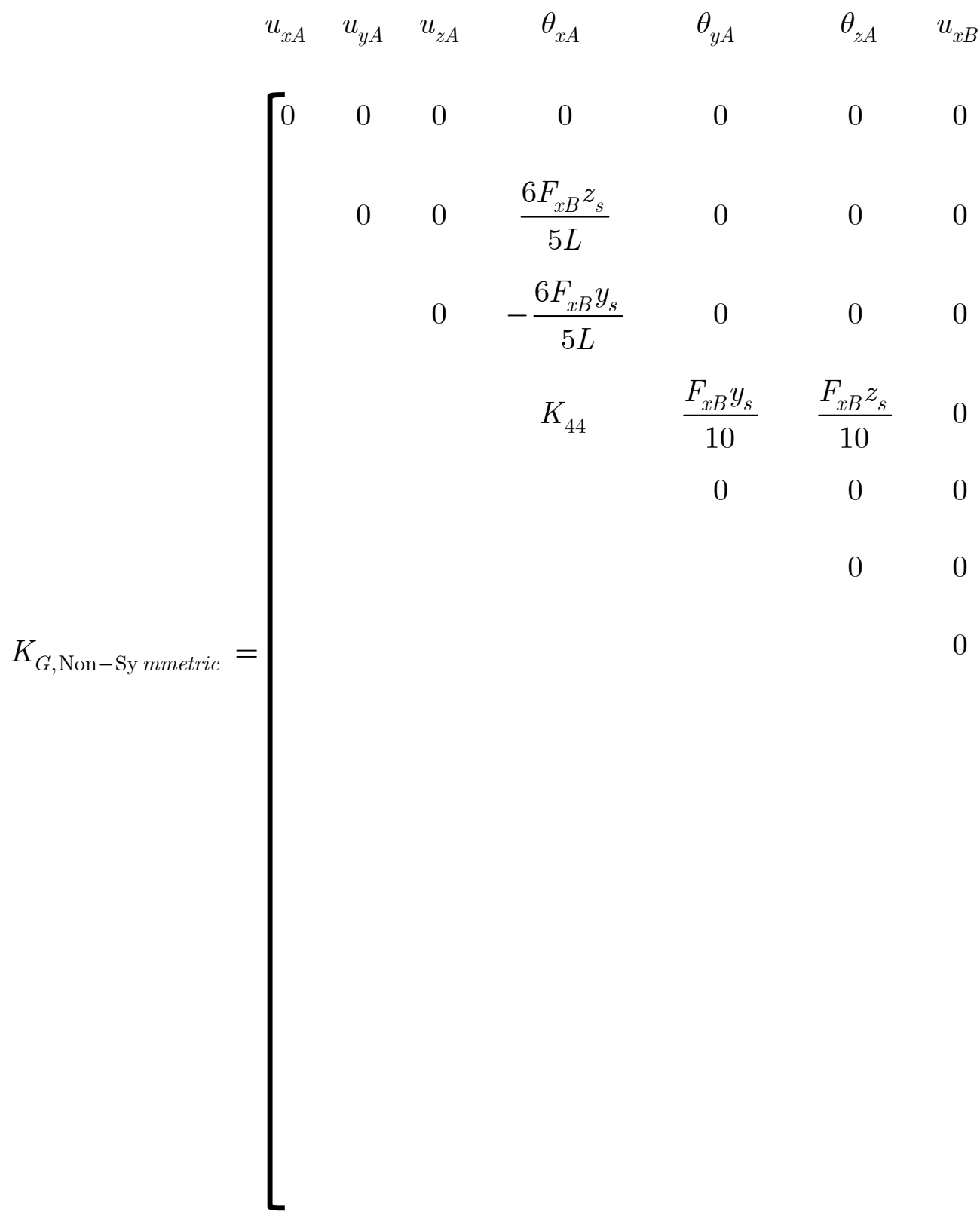

$k_{44}=\frac{-3 B_{A} \beta_{w}+3 B_{B} \beta_{w}-3 \beta_{y} M_{y A}+3 \beta_{y} M_{y B}+3 \beta_{z} M_{z A}-3 \beta_{z} M_{z B}-5 M_{z A y_{S}}-5 M_{z B y_{S}}+5 M_{y A z_{S}}+5 M_{y B z_{S}}}{5 L}$ 


$$
\begin{aligned}
& \begin{array}{lllllll}
u_{y B} & u_{z B} & \theta_{x B} & \theta_{y B} & \theta_{z B} & \theta_{x A}^{\prime} & \theta_{x B}^{\prime}
\end{array} \\
& \begin{array}{lllllll}
0 & 0 & 0 & 0 & 0 & 0 & 0
\end{array} \\
& 0 \quad 0 \quad-\frac{6 F_{x B} z_{s}}{5 L} \quad 0 \quad 0 \quad \frac{F_{x B} z_{s}}{10} \quad \frac{F_{x B} z_{s}}{10} \\
& 0 \quad 0 \quad \frac{6 F_{x B} y_{s}}{5 L} \quad 0 \quad 0 \quad 0 \quad-\frac{F_{x B} y_{s}}{10}-\frac{F_{x B} y_{s}}{10} \\
& -\frac{6 F_{x B} z_{s}}{5 L} \quad \frac{6 F_{x B} y_{s}}{5 L} \quad K_{4,10} \quad \frac{F_{x B} y_{s}}{10} \quad \frac{F_{x B} z_{s}}{10} \quad K_{4,13} \quad K_{4,14} \\
& 0 \quad 0 \quad-\frac{\left(F_{x B} y_{s}\right)}{10} \quad 0 \quad 0 \quad \frac{2 F_{x B} L y_{s}}{15}-\frac{F_{x B} L y_{s}}{30} \\
& 0 \quad 0-\frac{F_{x B} z_{s}}{10} \quad 0 \quad 0 \quad \frac{2 F_{x B} L z_{s}}{15}-\frac{F_{x B} L z_{s}}{30} \\
& \begin{array}{lllllll}
0 & 0 & 0 & 0 & 0 & 0 & 0
\end{array} \\
& 0 \quad \begin{array}{llllll}
6 F_{x B} z_{s} & 0 & 0 & -\frac{F_{x B} z_{s}}{5 L} & -\frac{F_{x B} z_{s}}{10}
\end{array} \\
& 0-\frac{6 F_{x B} y_{s}}{5 L} \quad 0 \quad 0 \quad \frac{F_{x B} y_{s}}{10} \quad \frac{F_{x B} y_{s}}{10} \\
& K_{10,10} \quad-\frac{F_{x B} y_{s}}{10}-\frac{F_{x B} z_{s}}{10} \quad-K_{4,13} \quad-K_{4,14} \\
& 0 \quad 0 \quad-\frac{F_{x B} L y_{s}}{30} \quad \frac{2 F_{x B} L y_{s}}{15} \\
& \left.\begin{array}{cc}
0-\frac{F_{x B} L z_{s}}{30} & \frac{2 F_{x B} L z_{s}}{15} \\
K_{13,13} & K_{13,14} \\
& K_{14,14}
\end{array}\right] \\
& k_{4,10}=\frac{3\left(B_{A} \beta_{w}-B_{B} \beta_{w}+\beta_{y} M_{y A}-\beta_{y} M_{y B}-\beta_{z} M_{z A}+\beta_{z} M_{z B}\right)}{5 L} \\
& k_{4,13}=\frac{B_{B} \beta_{w}+\beta_{y} M_{y B}-\beta_{z} M_{z B}}{10} \\
& k_{4,14}=\frac{-B_{A} \beta_{w}-\beta_{y} M_{y A}+\beta_{z} M_{z A}}{10} \\
& k_{10,10}=\frac{-3 B_{A} \beta_{w}+3 B_{B} \beta_{w}-3 \beta_{y} M_{y A}+3 \beta_{y} M_{y B}+3 \beta_{z} M_{z A}-3 \beta_{z} M_{z B}+5 M_{z A y_{S}}+5 M_{z B y_{S}}-5 M_{y A z_{S}}-5 M_{y B z_{S}}}{5 L} \\
& k_{13,13}=\frac{1}{30} L\left(-3 B_{A} \beta_{w}+B_{B} \beta_{w}-3 \beta_{y} M_{y A}+\beta_{y} M_{y B}+3 \beta_{z} M_{z A}-\beta_{z} M_{z B}\right) \\
& k_{13,14}=\frac{1}{60} L\left(B_{A} \beta_{w}-B_{B} \beta_{w}+\beta_{y} M_{y A}-\beta_{y} M_{y B}-\beta_{z} M_{z A}+\beta_{z} M_{z B}\right) \\
& k_{14,14}=-\frac{1}{30} L\left(B_{A} \beta_{w}-3 B_{B} \beta_{w}+\beta_{y} M_{y A}-3 \beta_{y} M_{y B}-\beta_{z} M_{z A}+3 \beta_{z} M_{z B}\right)
\end{aligned}
$$

